\title{
Hydro-magnetic mixed convection flow in a lid-driven cavity with wavy bottom surface
}

\author{
Litan Kumar Saha ${ }^{1,}$, , Monotos Chandra Somadder ${ }^{2}$, Nepal Chandra Roy ${ }^{2}$ \\ ${ }^{1}$ Department of Applied Mathematics, University of Dhaka, Dhaka, Bangladesh \\ ${ }^{2}$ Department of Mathematics, University of Dhaka, Dhaka, Bangladesh
}

Email address:

lksaha.math@gmail.com (L. K. Saha)

To cite this article:

Litan Kumar Saha, Monotos Chandra Somadder, Nepal Chandra Roy. Hydro-Magnetic Mixed Convection Flow in a Lid-Driven Cavity with Wavy Bottom Surface. American Journal of Applied Mathematics. Special Issue: Fluid Flow and Heat Transfer Inside a Closed Domain. Vol. 3, No. 1-1, 2015, pp. 8-19. doi: 10.11648/j.ajam.s.2015030101.12

\begin{abstract}
Mixed convection flow in the presence of magnetic field is examined in a lid-driven cavity with wavy bottom surface. The magnetic field is applied in perpendicular direction to the cavity. Moreover, the cavity is heated from top while the bottom surface is taken as a wavy pattern. The vertical walls of the cavity are adiabatic. The governing equations have been solved by using Galerkin weighted residual method of finite element formulation. To uncover the flow patterns and heat transfer mechanisms within the cavity, the results are presented in terms of streamlines and isotherms for different Reynolds number, Grashof number, Hartmann number and number of undulations offered by the wavy bottom surface. Also the effects of these parameters are shown on the Local Nusselt number. It is observed that the wavy lid-driven cavity can be considered as an effective heat transfer mechanism in presence of magnetic field at larger wavy surface amplitudes and low Richardson numbers.
\end{abstract}

Keywords: Mixed Convection, Lid-Driven Cavity, Magneto-Hydrodynamics, Wavy Surface, Heat Transfer

\section{Introduction}

Mixed convection involving the combined effect of forced and natural convection has been the focus of research due to its occurrence in numerous technological, engineering, and natural applications such as: cooling of electronic devices, lubrication technologies, drying technologies, food processing, float glass production [1], flow and heat transfer in solar ponds [2], thermal-hydraulics of nuclear reactors [3], and dynamics of lakes [4]. To acquire a body of knowledge about many of these engineering problems, flow and heat transfer processes in the presence of mixed convection in an enclosure with regular and irregular surfaces have been extensively studied.

Khanafer et al. [5] numerically investigated the natural convection heat transfer inside a porous enclosure with a sinusoidal vertical wavy wall. The authors surveyed a good number of studies on natural convection inside a wavy-walled enclosure. It was observed, in general, that the flow and heat transfer processes are significantly influenced by the roughened surface. Al-Amiri and Khanafer [6] analyzed the steady laminar mixed convection heat transfer in a lid-driven cavity with a flexible bottom surface. Since the machining geometries with wavy surfaces often experience difficulties, they proposed the use of flexible surfaces with known elasticity to enhance heat transfer characteristics. With a view to illustrating the phenomena, they considered a lid-driven cavity owing to its fundamental nature. They found that the flexible bottom wall along with the momentum and energy transport processes within the cavity greatly affects the flow and heat transfer mechanisms. Mendu and Das [7] used the lattice Boltzmann method to study the fluid flow in a cavity driven by an oscillating lid. Through a complete review of lid-driven flow in a cavity, they noted that the periodic nature of fluid flow in a cavity has not been thoroughly investigated yet. Thus it can be anticipated that there are some uncovered physics occurring in the relevant engineering problems. Oztop and Dagtekin [8] examined the steady state two-dimensional mixed convection problem in a vertical lid-driven square cavity of which the left and right moving walls are maintained at different constant temperatures and the bottom walls are adiabatic. They numerically found that both Richardson number and direction of moving walls affect the fluid flow and heat transfer in the cavity. Recently, Saha et al. [9] have performed the numerical simulation of the mixed convection flow and heat transfer in a lid-driven cavity with wavy bottom 
surface. They observed that the heat transfer mechanisms and flow characteristics inside the cavity noticeably depend on the number of undulations, Grashof number and Reynolds number.

Moreover, the literature review suggests that the flow behavior and heat transfer characteristics inside a cavity are changed not only for the surface geometry but also due to the presence of magnetic field. When an electromagnetic field exists in a fluid-filled cavity, then the corresponding problem of mixed convection becomes complex because of the shear flow generated by the movement of a wall and buoyancy induced flow. These types of problems have been investigated earlier for different thermal and flow boundary conditions such as two-sided lid driven cavities, one sided lid-driven cavities from top, bottom or vertical walls, oscillating walls, fully, partially or non-isothermally heated walls. The combined effect of natural and forced convection flow of an electrically conducting fluid in a cavity in the presence of magnetic field has attracted attention of researchers as it is often encountered in many industrial applications such as geothermal reservoirs, cooling of nuclear reactors, thermal insulations and petroleum reservoirs. In addition to, the magneto-hydrodynamic (MHD) effect is seen in electronic packages and microelectronic devices during their operations.

Most of the MHD studies have discussed on natural convection while a few number of studies focused on mixed convection in lid-driven cavities in the presence of magnetic field. Chamkha [10] carried out a numerical simulation using finite-volume approach of an unsteady, laminar, combined forced-free convection flow in a square cavity with internal heat generation and absorption and a magnetic field. When there is an internal heat generation source or sink inside the cavity, the average Nusselt number decreases for both aiding and opposing flow. A steady mixed convection flow and heat transfer in an obstructed lid-driven square cavity considering the conjugate effect of joule heating, magnetic field and heat-conducting obstacle operating under laminar regime has been investigated by Rahman et al. [11]. They found that the strength of the magnetic field significantly affects the fluid flow and heat transfer characteristics inside the cavity. Rudraiah et al. [12] investigated the effect of surface tension on buoyancy driven flow of an electrically conducting fluid in a rectangular cavity in the presence of a vertical transverse magnetic field to see how this force damps hydrodynamic movements. Sivasankaran et al. [13] numerically examined the mixed convection in a lid-driven cavity with sinusoidal boundary conditions at the sidewalls with magnetic field. They observed that the flow behavior and heat transfer rate inside the cavity strongly depend on the magnetic field.

From the above literature review, it can be concluded that the flow behavior and heat transfer mechanism can be characterized by the surface geometry of an enclosure, different thermal and flow boundary conditions and the presence of magnetic field. As the irregular surfaces and magnetic field have practical importance in engineering fields, the present study concerns the mixed convection lid-driven cavity flow with wavy bottom surface. In addition to, we consider the magnetic field in perpendicular direction to the cavity while the previous studies applied the magnetic field only in the direction parallel to $x$ or $y$. The cavity is sustained under a vertical temperature gradient by subjecting the top lid to a relatively higher temperature than the wavy bottom surface. The results are shown in terms of streamlines and isotherms for various pertinent parameters. These dimensionless parameters include the Reynolds number, Hartmann number and number of undulations offered by the wavy bottom surface. Finally, the effects of the above parameters are also depicted on the Local Nusselt number.

\section{Mathematical Formulation}

A square cavity of height and length $L$ with sinusoidal bottom surface, as shown in Figure 1, is considered field with fluid material initially at upper temperature than the fluid melting temperature. The upper wall of the cavity is moving with a constant velocity $U_{0}$ from left to right and the other wall has no-sleep condition. The bottom wall is kept at a low temperature, $T_{c}$, and the upper wall is kept at a high temperature, $T_{h}$. The free left wall and the right wall are considered to be adiabatic.

The assumptions made in this study are summarized here. The cavity is filled with an incompressible and electrically conducting fluid and a uniform magnetic field $B$ is applied perpendicular to the cavity. The cavity material is assumed to be Newtonian and incompressible, unsteady and laminar flow, no internal heat generation or absorption. In most buoyancy-driven convection problems, flow is generated by a temperature variation in the fluid system, which leads to local density differences. Under this consideration, a body force term has to be included into the momentum equations for the effect of local density differences. For temperature-driven flows, the Boussinesq approximation is often employed, that is, $g\left(\rho-\rho_{\mathrm{c}}\right)=g \beta\left(T-T_{c}\right)$ where $g$ is the acceleration due to gravity, $\beta$ is the coefficient of thermal expansion and $\rho$ is the fluid density. The above body force term is added to the momentum equation in the gravity direction.

In the low $R e_{m}$ limit, as is the case here, MHD effects exist only in the form of the Lorentz force, which is created by the combined action of the electric current, $J$, and magnetic field, $B$. The Lorentz and the buoyancy forces are the only external forces considered in this study.

The Lorentz force, $F$, is a result of the magnetic field effects on the electrically conducting fluid and is determined from the following equations:

$$
\begin{gathered}
\boldsymbol{F}=\frac{1}{\rho}(\boldsymbol{J} \times \boldsymbol{B}) \\
\boldsymbol{J}=\sigma(-\nabla \varphi+\boldsymbol{u} \times \boldsymbol{B}) \\
\nabla . \boldsymbol{J}=0 \\
\nabla . \boldsymbol{B}=0
\end{gathered}
$$


where $u=(u, v, 0)$ is the velocity vector, $B=\left(0,0, B_{0}\right)$ is the total magnetic field vector, $J$ is the electric current density, $\sigma$ is the electrical conductivity, $\nabla$ is the nabla operator, $\nabla$ is the electrostatic potential and $J=\sigma(-\nabla \varphi+u \times B)$ is the Ohm's law. At low $R e_{m}$, the flow is affected by the magnetic field, but the effect of the flow on the magnetic field is negligible. Consequently, the Lorentz force term, $(J \times B) / \rho$ in equation (1) is a function of only $B$ and $u$.

Now applying vector calculus, equations (1) and (2) give

$$
F=-\frac{\sigma B_{0}{ }^{2}}{\rho} u-\frac{\sigma B_{0}{ }^{2}}{\rho} v
$$

Therefore, in such flows, a body force term needs to be added to the momentum equations to include the effect of magnetic force.

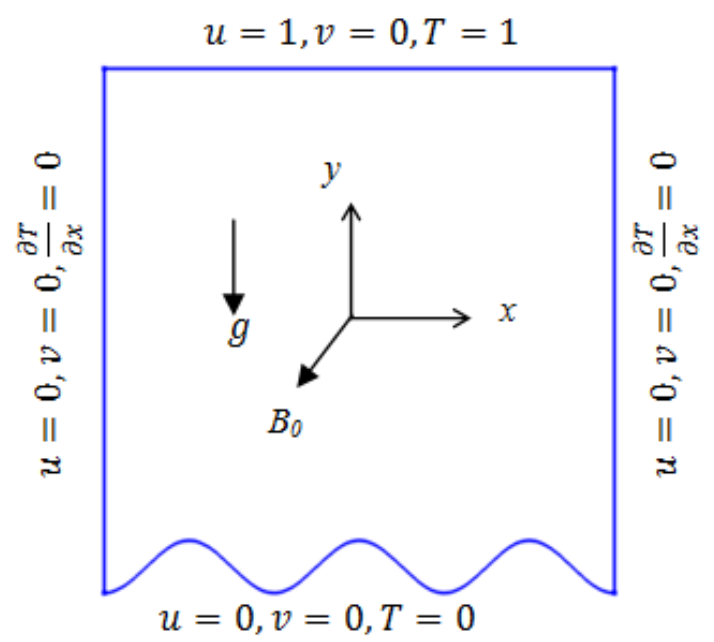

Figure 1. Cavity geometry and boundary condition.

Thus, the steady mass, momentum and energy conservation equation becomes:

Mass conservation equation:

$$
\frac{\partial u}{\partial x}+\frac{\partial v}{\partial y}=0
$$

Momentum conservation equation:

$$
\begin{gathered}
u \frac{\partial u}{\partial x}+v \frac{\partial u}{\partial y}=-\frac{1}{\rho} \frac{\partial p}{\partial x}+v\left(\frac{\partial^{2} u}{\partial x^{2}}+\frac{\partial^{2} u}{\partial y^{2}}\right)-\frac{\sigma B_{0}^{2}}{\rho} u \\
u \frac{\partial v}{\partial x}+v \frac{\partial v}{\partial y}=-\frac{1}{\rho} \frac{\partial p}{\partial y}+v\left(\frac{\partial^{2} v}{\partial x^{2}}+\frac{\partial^{2} v}{\partial y^{2}}\right) \\
+g \beta\left(T-T_{c}\right)-\frac{\sigma B_{0}^{2}}{\rho} v
\end{gathered}
$$

Energy conservation equation:

$$
u \frac{\partial T}{\partial x}+v \frac{\partial T}{\partial y}=\alpha\left(\frac{\partial^{2} T}{\partial x^{2}}+\frac{\partial^{2} T}{\partial y^{2}}\right)
$$

Here $u$ and $v$ are the $x$ - and $y$-components of the velocity field, $p$ is the pressure, $T$ is the temperature, $\beta$ is the volumetric thermal expansion coefficient, $\alpha$ is the thermal diffusivity, $\sigma$ is the electrical conductivity and $B_{0}$ is the applied constant magnetic force in perpendicular direction to the cavity.

The following non-dimensional scales are adopted for mixed convection in the absence of a reference velocity value:

$$
\begin{gathered}
X=\frac{x}{L}, Y=\frac{y}{L}, U=\frac{u}{U_{0}}, V=\frac{v}{U_{0}}, \\
P=\frac{p}{\rho U_{0}^{2}}, \theta=\frac{T-T_{c}}{T_{h}-T_{c}}
\end{gathered}
$$

Introducing the above non-dimensional scales into the governing equations, we obtain the non-dimensional form of the equations as follows:

$$
\begin{gathered}
\frac{\partial U}{\partial X}+\frac{\partial U}{\partial Y}=0 \\
U \frac{\partial U}{\partial X}+V \frac{\partial U}{\partial Y}=-\frac{\partial P}{\partial X} \\
+\frac{1}{\operatorname{Re}}\left(\frac{\partial^{2} U}{\partial X^{2}}+\frac{\partial^{2} U}{\partial Y^{2}}\right)-\frac{H a^{2}}{\operatorname{Re}} U \\
U \frac{\partial V}{\partial X}+V \frac{\partial V}{\partial Y}=-\frac{\partial P}{\partial Y} \\
+\frac{1}{\operatorname{Re}}\left(\frac{\partial^{2} V}{\partial X^{2}}+\frac{\partial^{2} V}{\partial Y^{2}}\right)+\frac{G r}{\operatorname{Re}^{2}} \theta-\frac{H a^{2}}{\operatorname{Re}} V \\
U \frac{\partial \theta}{\partial X}+V \frac{\partial \theta}{\partial Y}=\frac{1}{\operatorname{Re} \operatorname{Pr}}\left(\frac{\partial^{2} \theta}{\partial X^{2}}+\frac{\partial^{2} \theta}{\partial Y^{2}}\right)
\end{gathered}
$$

In the above equations $\operatorname{Re}=U_{0} L / v, \operatorname{Pr}=v / \alpha, G r=g \beta \Delta T L^{3} / v^{2}$ are respectively the non-dimensional Reynolds number, Prandtl number and Grashof number.

$\mathrm{Ha}$ is the Hartman number, is a dimensionless parameter, defined as the square root of ratio of electromagnetic force to viscous force.

$$
H a=B_{0} L \sqrt{\frac{\sigma \rho}{v}}
$$

The shape of the bottom wavy surface profile is assumed to mock the following pattern $Y=A(1-\cos (2 \lambda \pi X))$ where $A$ is the dimensionless amplitude of the wavy surface and $\lambda$ is the number of undulations.

The dimensionless boundary condition becomes:

$$
\begin{aligned}
& U=1, V=0, \theta=1 \quad \text { for } \quad 0 \leq X \leq 1, Y=1 \\
& U=V=0, \theta=0 \text { for } 0 \leq X \leq 1, Y=A(1-\cos (2 \lambda \pi X)) \\
& U=V=0, \frac{\partial \theta}{\partial X}=0 \text { for } 0 \leq Y \leq 1, X=0, X=1 .
\end{aligned}
$$

Since the convective heat transfer coefficient, $h$, and Nusselt number, $N u$, depend on the temperature gradient at a flat surface, $(-\partial T / \partial n)$ where $n$ is normal direction to the 
surface. Therefore, we can obtain the rate of heat flux from each of the walls. The corresponding average Nusselt number, for the heated wall, is defined as:
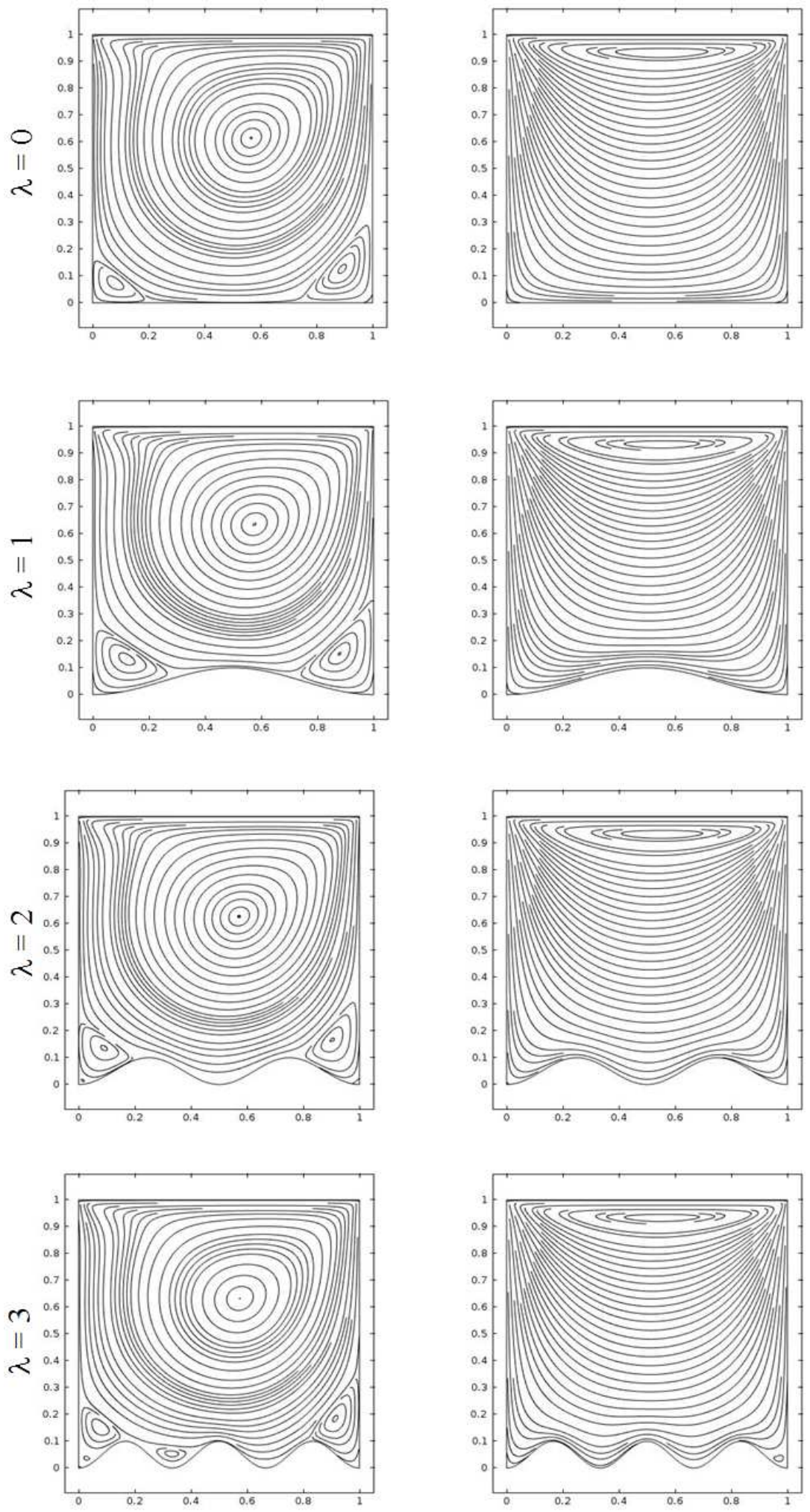

(a) $H a=0$

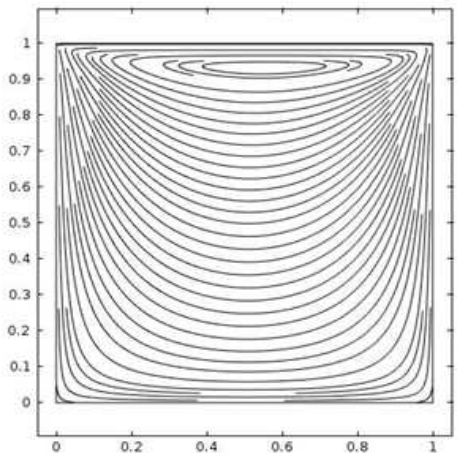

$$
N u_{a v}=\frac{1}{S} \int_{0}^{s} N u d x
$$

where $S$ is the total chord length of the wavy surface and $s$ is the coordinate along the wave surface.
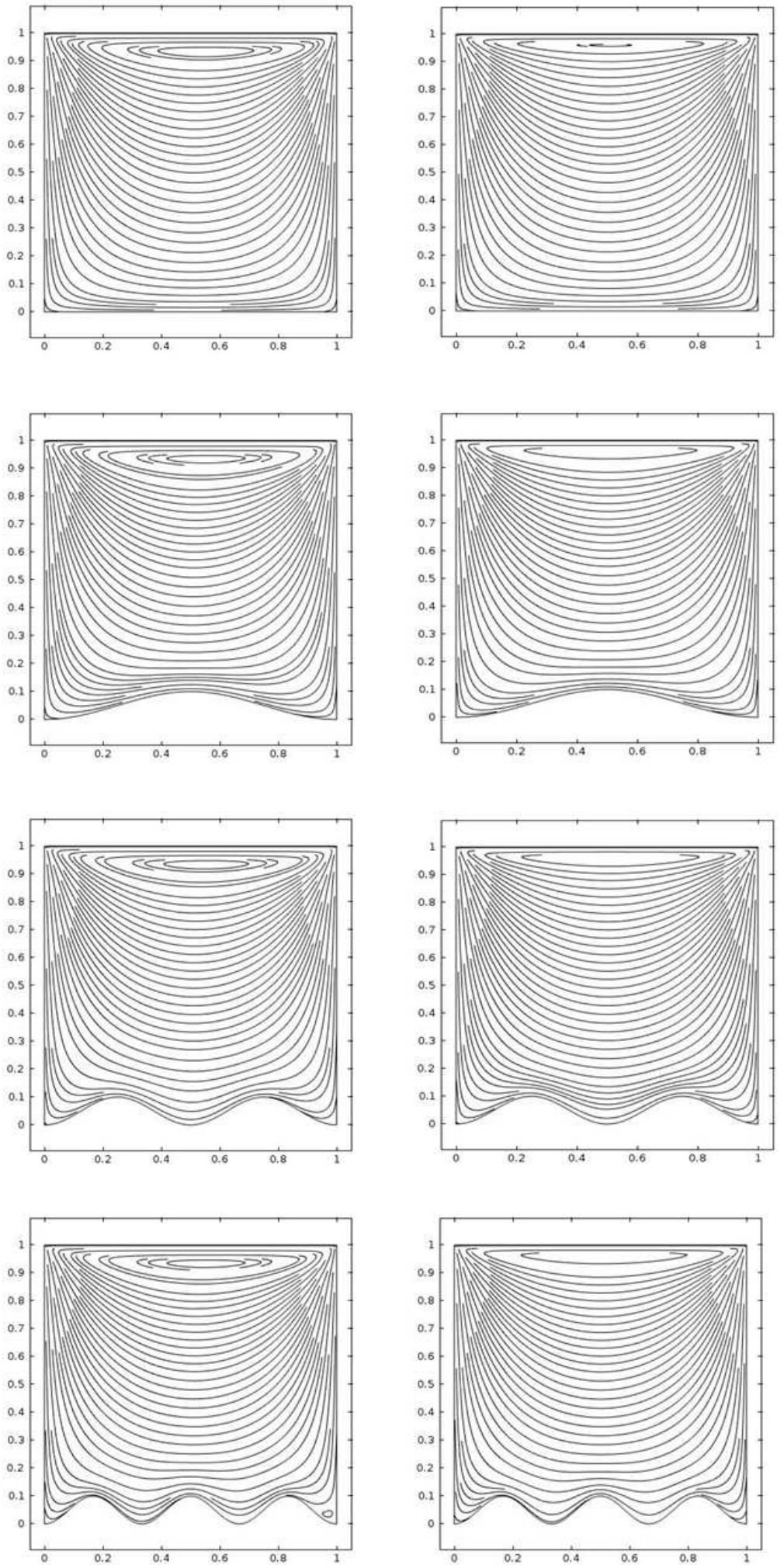

(b) $\mathrm{Ha}=50$

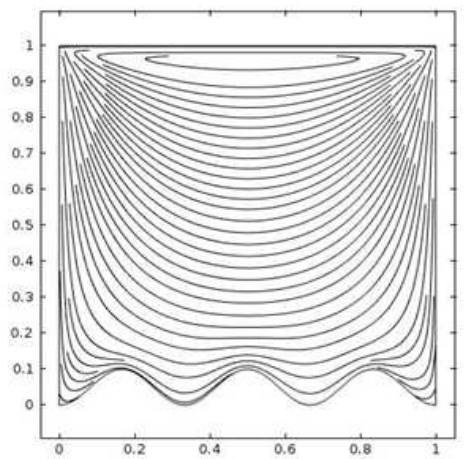

(c) $H a=100$

Figure 2. Stream line contour for various undulations at $\mathrm{Re}=400, \mathrm{Gr}=1 \times 10^{4}$ while (a) $\mathrm{Ha}=0$ (b) $\mathrm{Ha}=50$ (c) $\mathrm{Ha}=100$. 

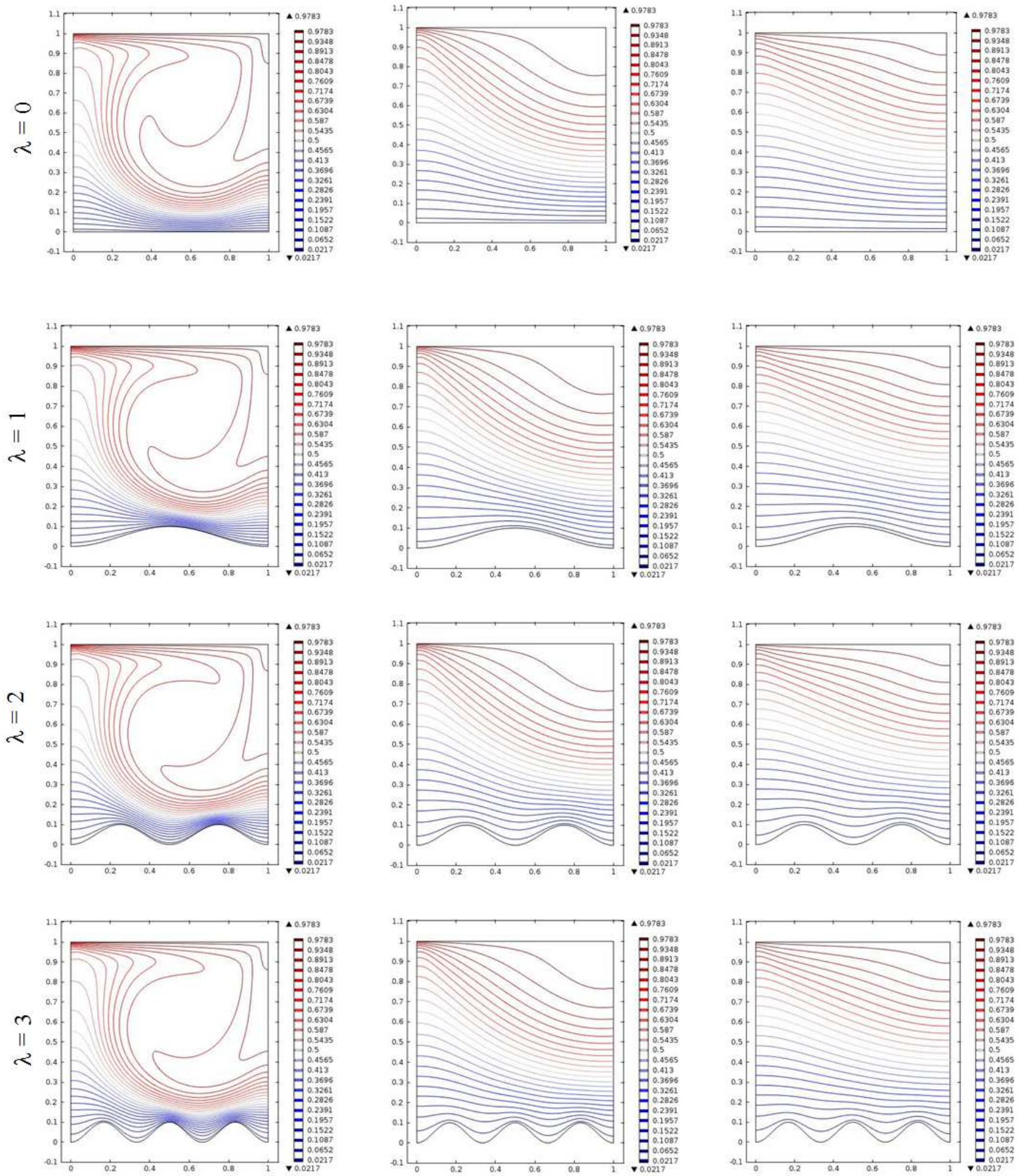

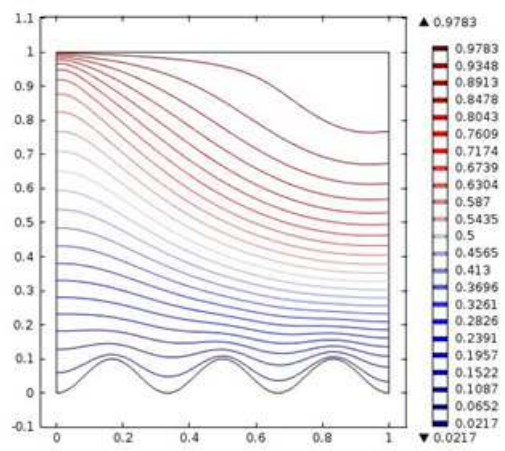

(b) $H a=50$

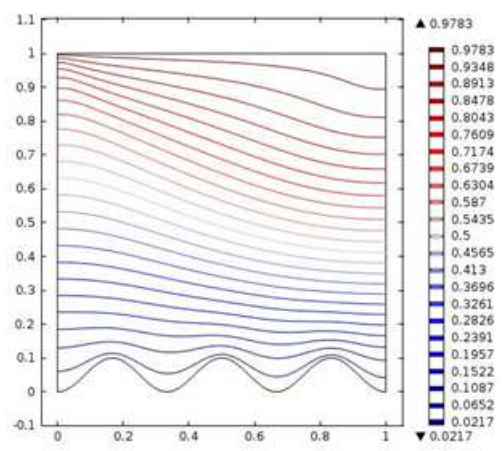

(c) $\mathrm{Ha}=100$

Figure 3. Isothermal contour for various undulations at for $\mathrm{Re}=400, \mathrm{Gr}=1 \times 10^{4}$ while (a) $\mathrm{Ha}=0$ (b) $\mathrm{Ha}=50$ (c) $\mathrm{Ha}=100$. 

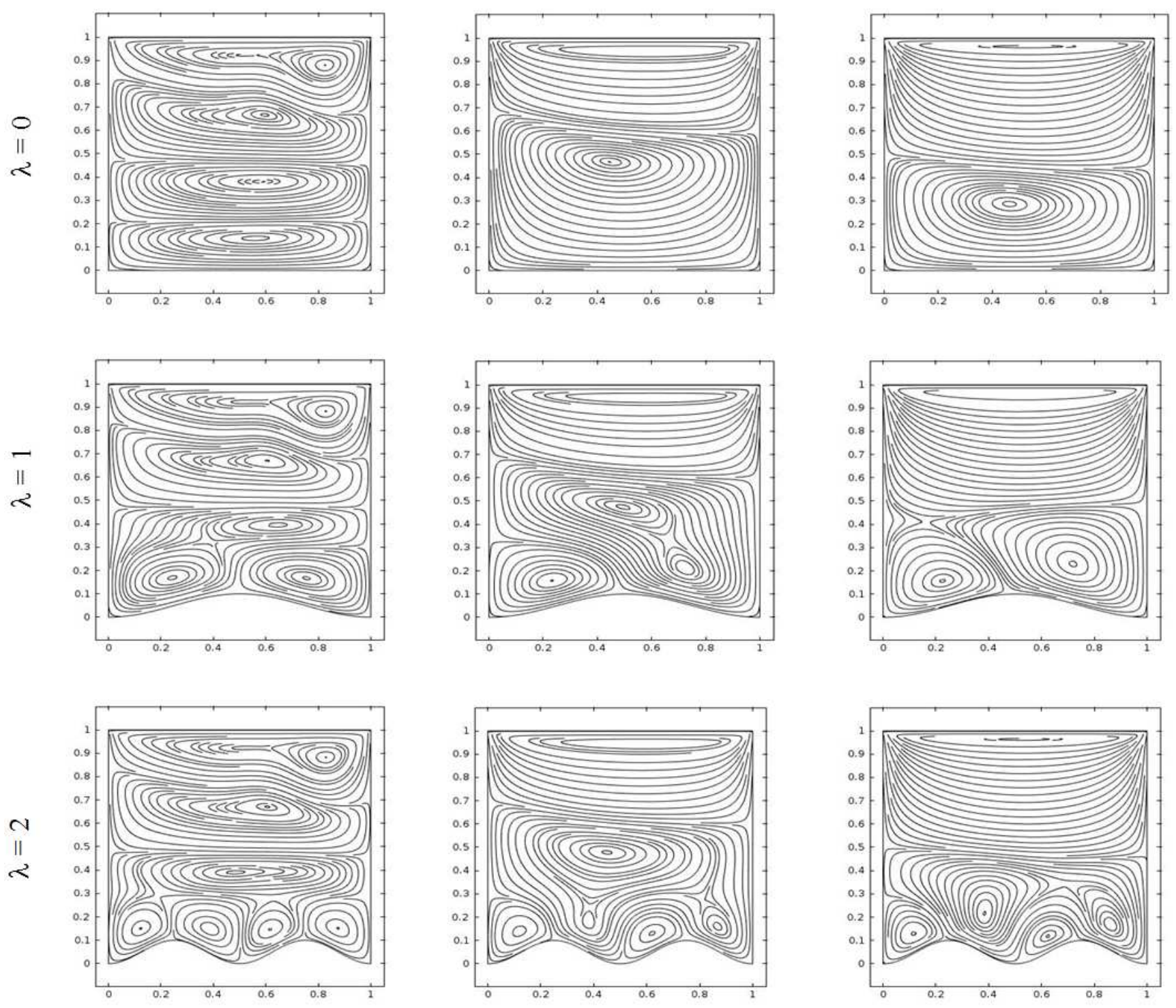

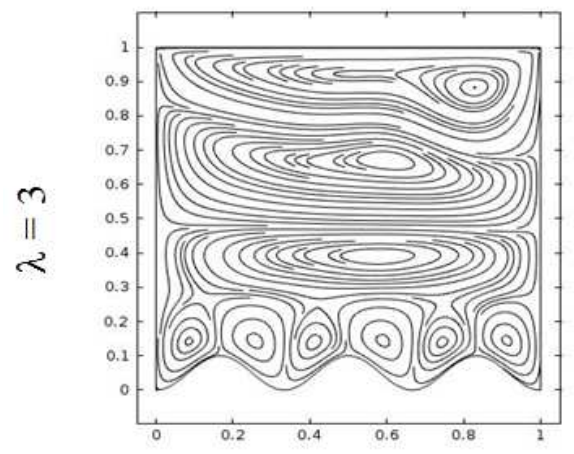

(a) $\mathrm{Ha}=0$

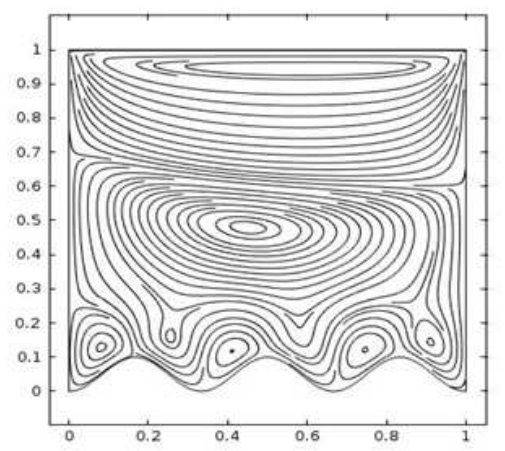

(b) $H a=50$

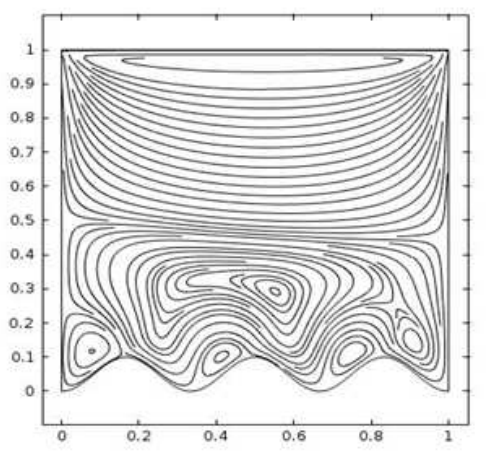

(c) $\mathrm{Ha}=100$

Figure 4. Stream line contour for various undulations at $\mathrm{Re}=400, \mathrm{Gr}=1 \times 10^{6}$ while (a) $\mathrm{Ha}=0$ (b) $\mathrm{Ha}=50$ (c) $\mathrm{Ha}=100$. 

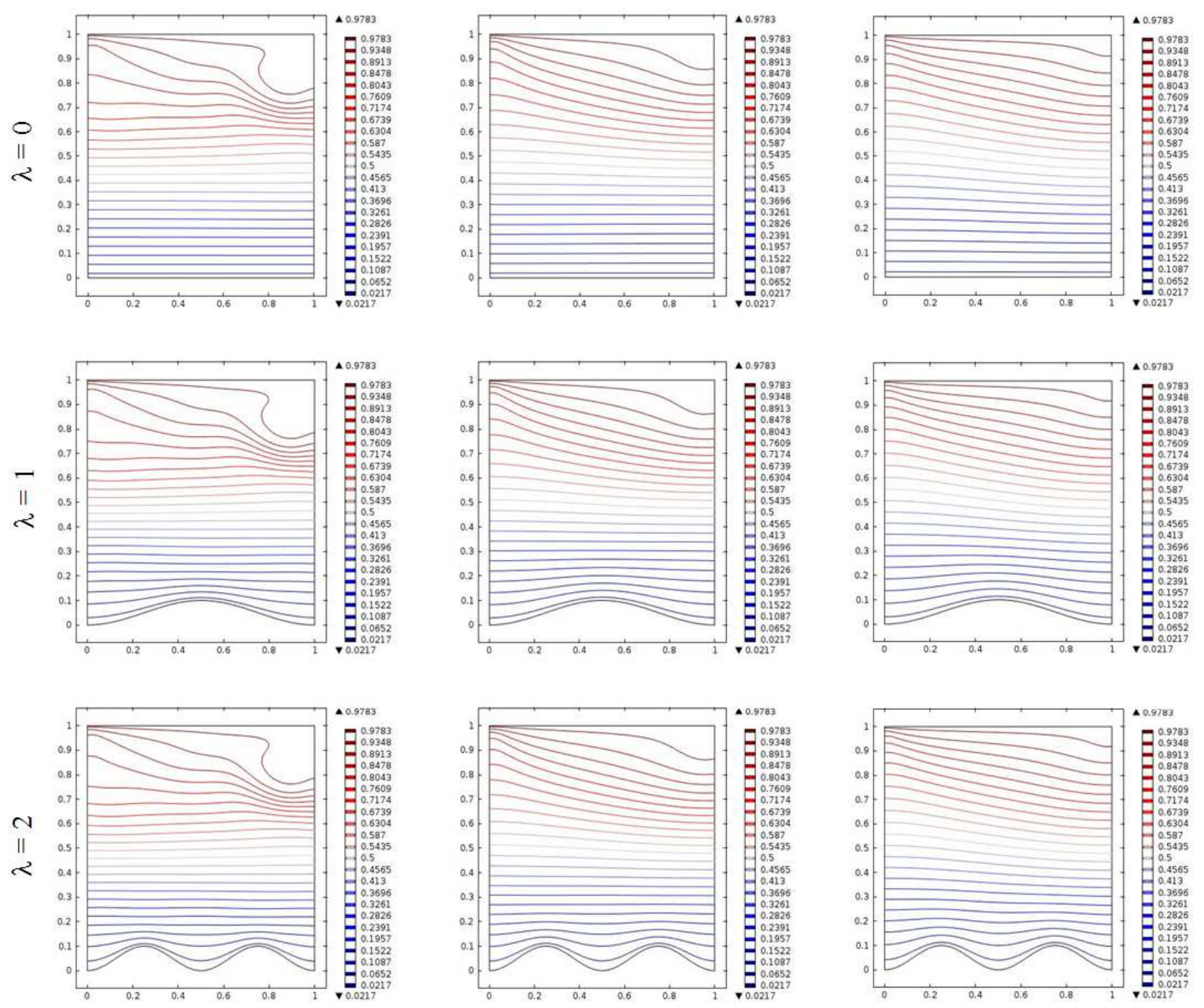

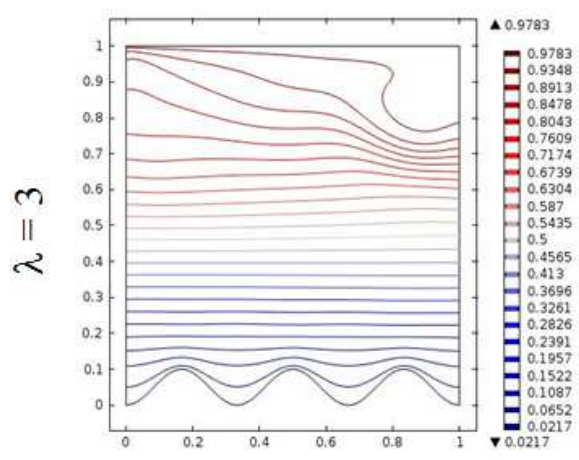

(a) $H a=0$

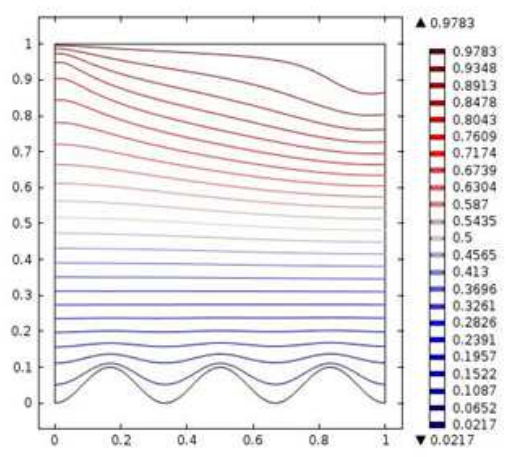

(b) $H a=50$

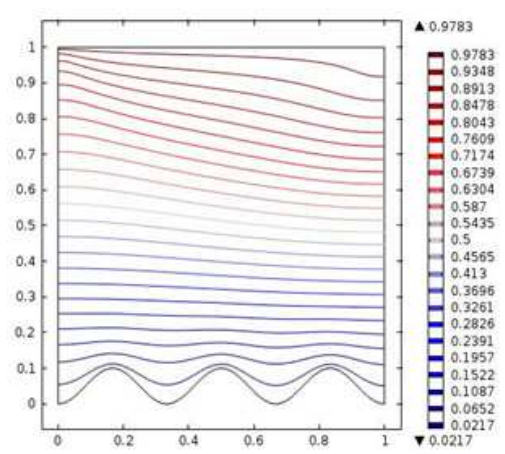

(c) $\mathrm{Ha}=100$

Figure 5. Isothermal contour for various undulations at $\mathrm{Re}=400, \mathrm{Gr}=1 \times 10^{6}$ (a) $\mathrm{Ha}=0$ (b) $\mathrm{Ha}=50$ (c) $\mathrm{Ha}=100$. 

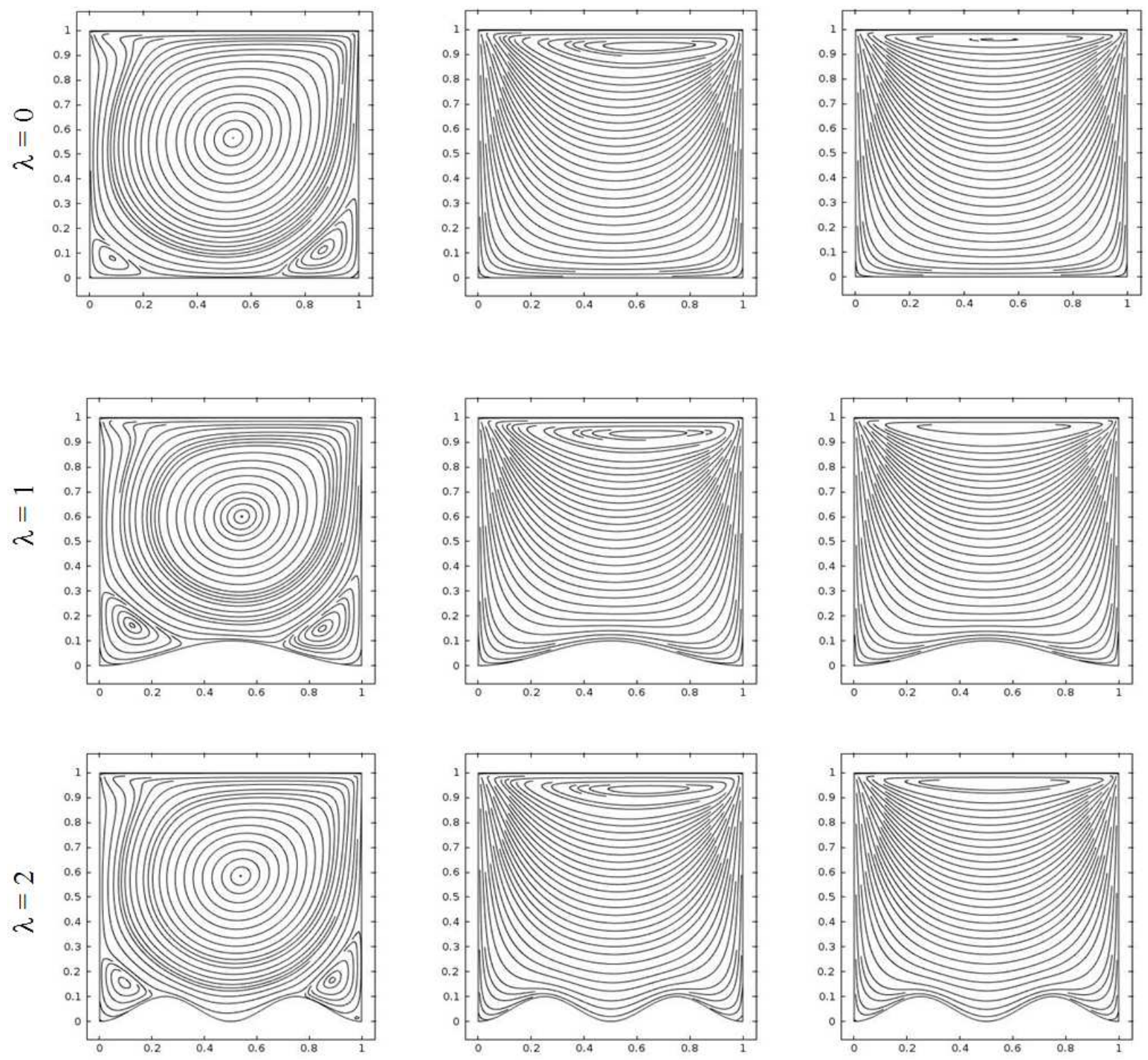

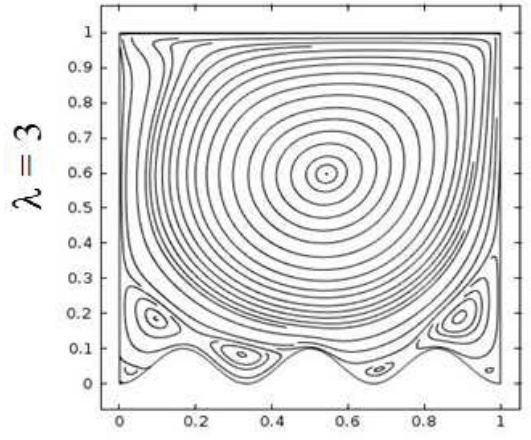

(a) $\mathrm{Ha}=0$

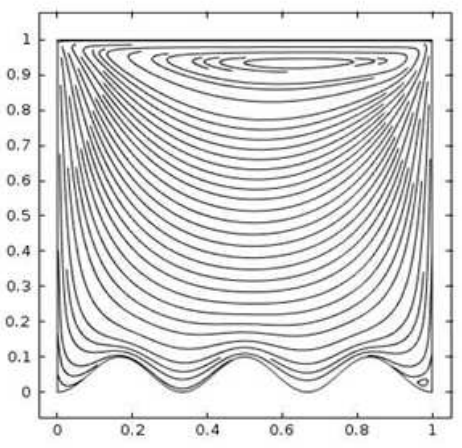

(b) $\mathrm{Ha}=50$

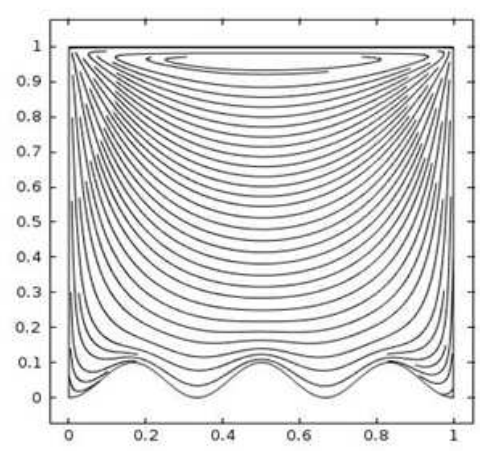

(c) $\mathrm{Ha}=100$

Figure 6. Stream line contour for various undulations at $\mathrm{Gr}=1 \times 10^{4}, \mathrm{Re}=1 \times 10^{3}$ while (a) $\mathrm{Ha}=0$ (b) $\mathrm{Ha}=50$ (c) $\mathrm{Ha}=100$. 

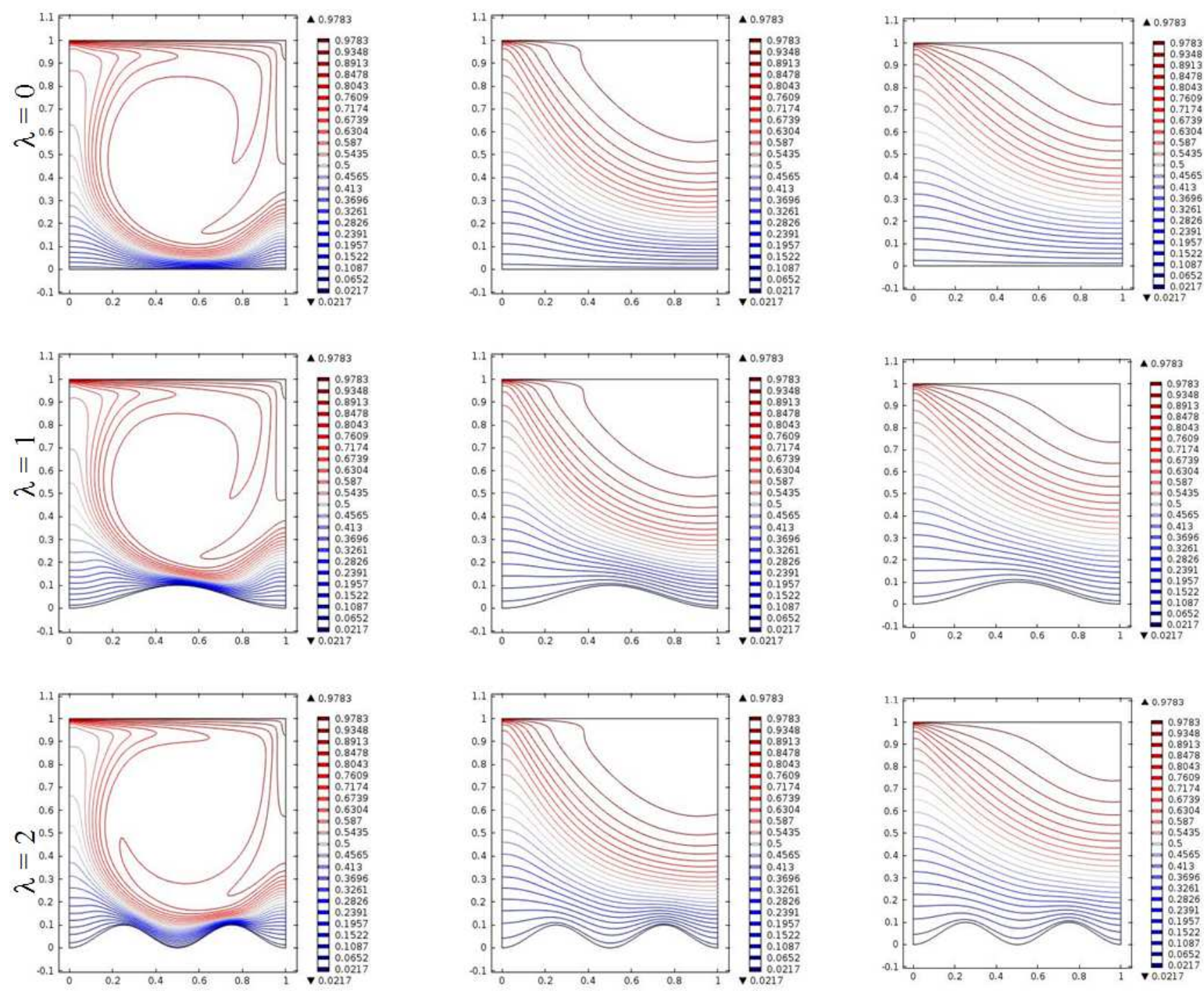

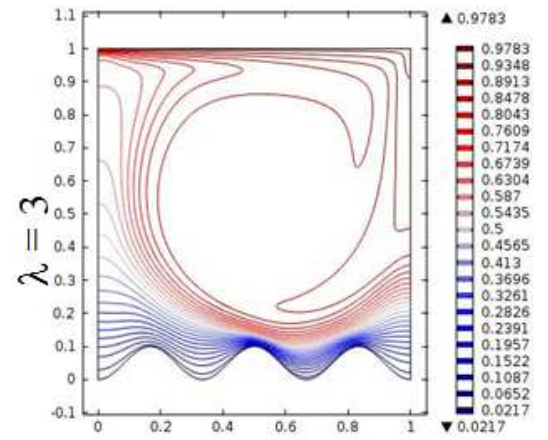

(a) $\mathrm{Ha}=0$

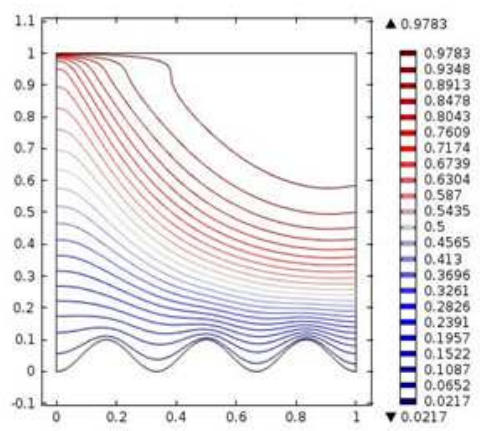

(a) $\mathrm{Ha}=50$

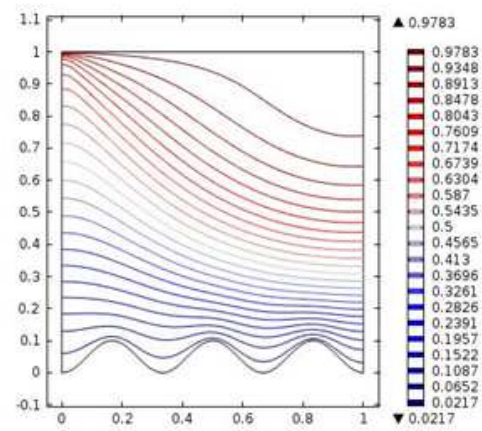

(a) $\mathrm{Ha}=100$

Figure 7. Isothermal contour for various undulations $\lambda$ at $\mathrm{Gr}=1 \times 10^{4}, \mathrm{Re}=1 \times 10^{3}$ while (a) $\mathrm{Ha}=0$ (b) $\mathrm{Ha}=50$ (c) $\mathrm{Ha}=100$. 


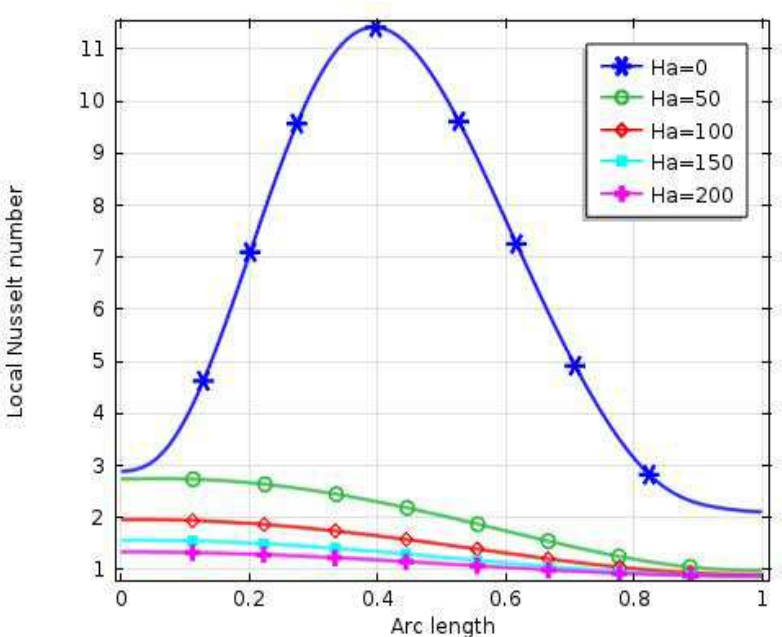

(a)

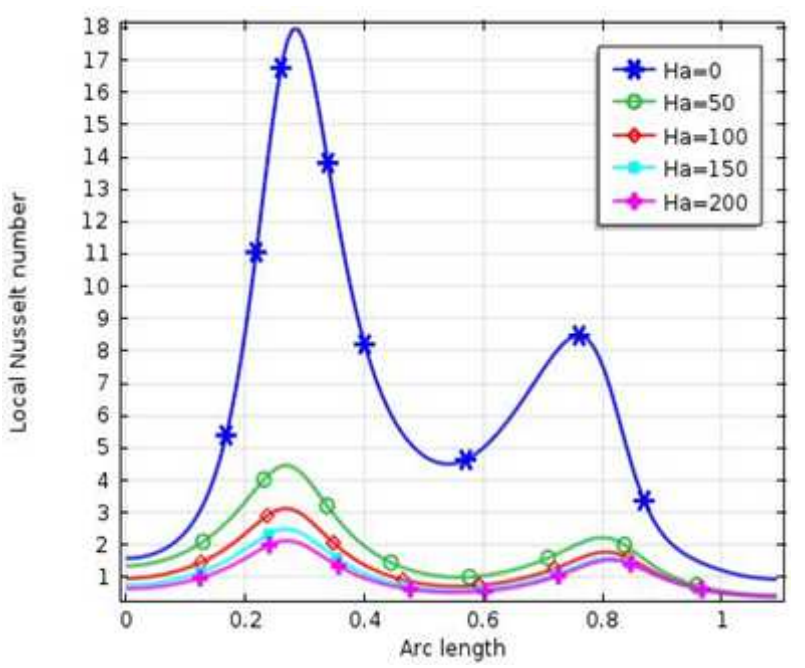

(c)

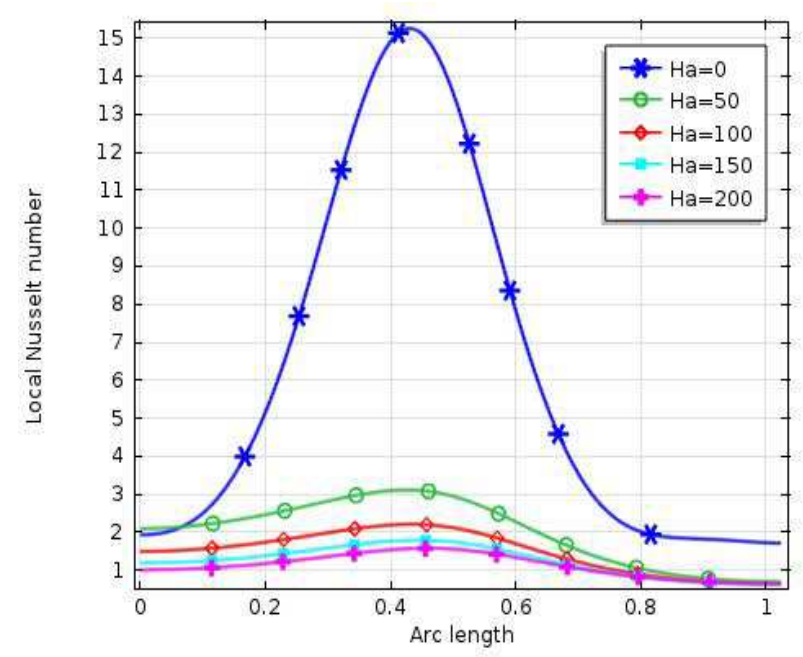

(b)

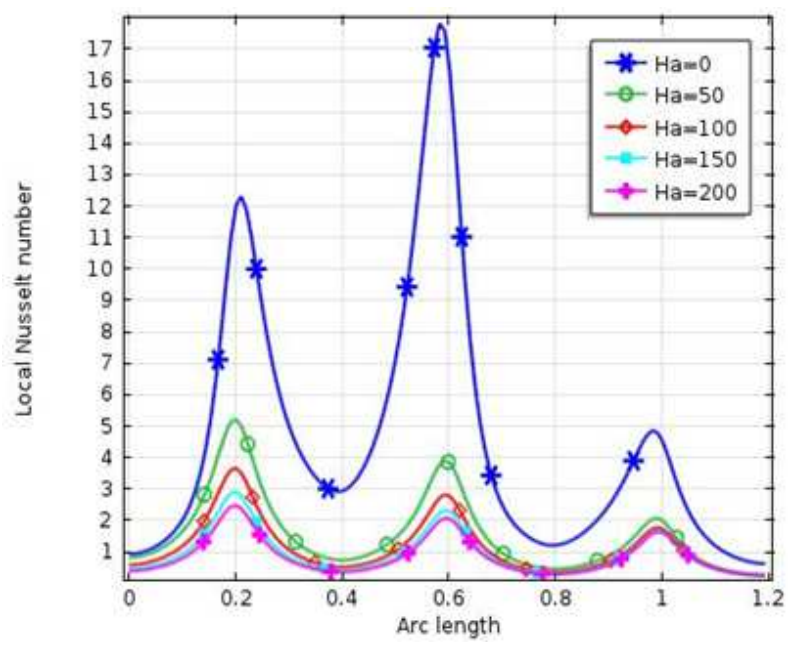

(d)

Figure 8. Variation of Local Nusselt number for various values of Ha at $\mathrm{Gr}=1 \times 10^{4}$, Re $=10^{3}$ while (a) $\lambda=0$ (b) $\lambda=1$ (c) $\lambda=2$ (d) $\lambda=3$.

\section{Results and Discussion}

The characteristics of the flow and temperature fields in the lid-driven cavity are examined by exploring the effects of the Richardson number $R i$ (ratio of the Grashof number and square of the Reynolds number), the Hartmann number $\mathrm{Ha}$ and number of undulations $\lambda$. Such field variables are examined by outlaying the steady state version of the streamlines and temperature distributions as well as the Local Nusselt number $\mathrm{Nu}$.

The impact of varying the Hartman number $H a$ for fixed $R i$ $=G r / R e^{2}$ when $G r=1 \times 10^{4}$ and $R e=400$ on the streamlines for various number of undulations is gauged through the results illustrated in Figures 2(a)-(c). The Hartmann number presents a measure for the importance of the magnetic field acting on forced convection forces relative to the natural convection effect. For $\mathrm{Ha}=0$, a large rotating clockwise vortex is developed occupying the major portion of the cavity.
In addition, a minor vortex tends to form near the right bottom corner and left bottom corner. Moreover, the number of small vortex near the bottom surface increases with increasing $\lambda$. Furthermore, for a particular value of $\lambda$, the size of vortex decreases with elevating $\mathrm{Ha}$ due to the fact, that the magnetic field tends to retard the fluid motion in the bulk of the cavity.

The temperature field depending on $H a$ and $\lambda$ for corresponding value of Figures 2 is shown in Figures 3(a)-(c). For a fixed $H a$, the thermal field remains almost similar but the thermal boundary layer near the bottom surface becomes slightly compressed when the number of waves increases. Further, isothermal pattern changes dramatically with increasing $\mathrm{Ha}$ for a fixed $\lambda$.

The impact of varying the Hartman number $\mathrm{Ha}$ for fixed Ri $=\mathrm{Gr} / \mathrm{Re}^{2}$ when $\mathrm{Gr}=1 \times 10^{6}$ and $\mathrm{Re}=400$ on the streamlines for various number of undulations is gauged through the results illustrated in Figures 4(a)-(c). The Hartmann number presents a measure for the importance of the magnetic field 
acting on natural convection forces relative to the forced convection effect. For $\mathrm{Ha}=50$ and 100 near the bottom surface have formed various vortex of various form depending on the number of undulation because the magnetic field tends to retard the fluid motion in the bulk of the cavity.

The temperature field depending on $\mathrm{Ha}$ and $\lambda$ for corresponding value of Figures 4 is shown in Figures 5(a)-(c). For a fixed $H a$, the thermal field remains almost similar but the thermal boundary layer near the bottom surface becomes slightly compressed when the number of waves increases. Further, isothermal pattern changes dramatically with increasing $\mathrm{Ha}$ for a fixed $\lambda$.

The impact of varying the Hartman number $\mathrm{Ha}$ for fixed $\mathrm{Ri}$ $=G r / R^{2}$ when $G r=1 \times 10^{4}$, and $R e=1 \times 10^{3}$ on the streamlines for various numbers of undulations is gauged through the results illustrated in Figures 6(a)-(c). The Hartmann number presents a measure for the importance of the magnetic field for highly forced convection flow. The number of small vortex near the bottom surface increases with increasing $\lambda$. Furthermore, for a particular value of $\lambda$, the size of vortex decreases with elevating Ha due to the fact, that the magnetic field tends to retard the fluid motion in the bulk of the cavity.

The temperature field depending on $\mathrm{Ha}$ and $\lambda$ for corresponding value of Figures 6 is shown in Figures 7(a)-(c). For a fixed $\mathrm{Ha}$, the thermal field remains almost similar but the thermal boundary layer near the bottom surface becomes slightly compressed when the number of waves increases. Further, isothermal pattern changes dramatically with increasing Ha for a fixed $\lambda$.

The influences of the Hartmann number on the Local Nusselt number for various numbers of undulations $\lambda$ are graphically depicted in Figures 8(a)-(d). The results exhibit that the Local Nusselt number is found to increase for higher values of $\lambda$, and it has the maximum value for second undulation. As the value of $H a$ increases Local Nusselt number decreases which indicates that increasing value of $\mathrm{Ha}$ delays the flow in the bulk of the cavity and temperature gradient.

\section{Conclusion}

Hydrodynamic mixed convection heat transfer in a lid-driven cavity heated from top is studied numerically for various pertinent dimensionless parameters. The bottom surface is considered to follow a wavy pattern. Furthermore, the vertical walls were subjected to insulated boundary conditions. The governing equations are solved using the Galerkin finite element method. Effects of Reynolds number, Grashof number and number of undulations are highlighted to reveal their impacts on flow structure and heat transfer characteristics. The results show that the variation in the Reynolds number affects significantly the flow and thermal current activities. The increase in the ratio of Grashof number and square of Reynolds number (Richardson number) to obstruct flow and thermal current activities owing to the increase in the imposed vertical temperature gradient. The increase in the Hartmann number delays the flow and consequently the isothermal lines occupy almost the whole region of the cavity. An increase in the number of undulations at the wavy bottom wall causes small vortices near it while there is a large one occupying almost the whole cavity. In addition to, the thermal boundary layer becomes thick for any particular Re. On the other hand, the number of undulations at the bottom wall noticeably influences the flow and heat transfer characteristics inside the cavity for variation in $\mathrm{Ha}$. The results have also demonstrated that the average Nusselt number was found optimum using two undulations at low Richardson numbers, which can be attained by increasing the sliding lid speed. Finally, the local and average Nusselt number predictions are found to increase with an increase in the amplitude of the wavy surface while maintaining a relatively low Richardson number. Thus, the wavy lid-driven cavity can be considered as an effective heat transfer mechanism in presence of magnetic field at larger wavy surface amplitudes and low Richardson numbers.

\section{References}

[1] L. A. B. Pilkington, Review lecture: The Float Glass Process, third ed., in: Proc. R. Soc. Lond. A, Vol. 314, pp. 1-25 (1969).

[2] C. K. Cha, Y. Jaluria, Recirculating mixed convection flow for energy extraction, Int. J. Heat Mass Transfer, Vol. 27, pp. 1801-1810 (1984).

[3] F. J. K. Ideriah, Prediction of turbulent cavity flow driven by buoyancy and shear, J. Mech. Eng. Sci., Vol. 22, pp. 287-295 (1980).

[4] J. Imberger, P.F. Hamblin, Dynamics of lakes, reservoirs, and cooling ponds, Adv. Rev. Fluid Mech., Vol. 14, pp. 153-187 (1982).

[5] K. Khanafer, B. Al-Azmi, A. Marafie, I. Pop, Non-Darcian effects on natural convection heat transfer in a wavy porous enclosure, Int. J. Heat Mass Transfer, Vol. 52, pp. 1887-1896 (2009).

[6] A. Al-Amiri, K. Khanafer, Fluid-structure interaction analysis of mixed convection heat transfer in a lid-driven cavity with a flexible bottom wall, Int. J. Heat and Mass Transfer, Vol. 54, pp. 3826-3836 (2011).

[7] S. S. Mendu, P. K. Das, Fluid flow in a cavity driven by an oscillating lid-A simulation by lattice Boltzmann method, Eur. J. Mech. B Fluids, Vol. 39, 59-70 (2013).

[8] H. F. Oztop, I. Dagtekin, Mixed convection in two-sided lid-driven differentially heated square cavity, Int. J. Heat Mass Transfer, Vol. 49, pp. 1761-1769 (2004).

[9] L. K. Saha, M. C. Somadder, K. M. S. Uddin, Mixed convection heat transfer in a lid driven cavity with wavy bottom surface, Amer. J. Appl. Math., Vol. 1(5), 92-101 (2013).

[10] A. J. Chamkha, Hydromagnetic combined convection flow in a vertical lid-driven cavity with internal heat generation or absorption, Num. Heat Transfer, Part A, Vol. 41, pp. 529-546 (2002). 
[11] M. M. Rahman, M. A. Alim, M. M. A. Sarker, Numerical study on the conjugate effect of joule heating and magnato-hydrodynamics mixed convection in an obstructed lid-driven square cavity, Int. Comm. Heat Mass Transfer, Vol. 37 (5), pp. 524-534 (2010).

[12] N. Rudraiah, M. Venkatachalappa, C. K. Subbaraya, Combined surface tension and buoyancy-driven convection in a rectangular open cavity in the presence of magnetic field, Int. J. Non-Linear Mech., Vol. 30 (5), pp. 759-770 (1995).
[13] S. Sivasankaran, A. Malleswaran, J. Lee, P. Sundar, Hydro-magnetic combined convection in a lid-driven cavity with sinusoidal boundary conditions on both sidewalls, Int. J. Heat Mass Transfer, Vol. 54, 512-525 (2011).

[14] A. Al-Amiri, K. Khanafer, I. Pop, Numerical simulation of unsteady mixed convection in a driven cavity using an externally excited sliding lid, Int. J. Heat Mass Transfer, Vol. 50, pp. 1771-1780 (2007). 